\title{
MINIMAL PRIMES OF IDEALS AND INTEGRAL RING EXTENSIONS
}

\author{
WILLIAM HEINZER ${ }^{1}$
}

\begin{abstract}
It is shown that if $R$ is a commutative ring with identity having the property that ideals in $R$ have only a finite number of minimal primes, then a finite $R$-algebra again has this property. It is also shown that an almost finite integral extension of a noetherian integral domain has noetherian prime spectrum.
\end{abstract}

If $\mathfrak{a}$ is an ideal in a ring $R$ (tacitly assumed to be commutative with identity) and $P$ is a prime ideal of $R$ containing a, then $P$ is called a minimal prime of $a$ if there is no prime ideal of $R$ containing $a$ and properly contained in $P$. The ring $R$ is said to have FC (for finite components) if each ideal of $R$ has only a finite number of minimal primes.

I am indebted to Professor Nagata for suggestions which helped me in obtaining the proofs of the theorems in this article, and I would like to thank him for his generous help.

The special case in Theorem 1 where $R$ is an integrally closed domain was proved in [1, Corollary 11, p. 577].

THEOREM 1. If $R$ is a ring with $F C$ and $R^{\prime}$ is a finite $R$-algebra, then $R^{\prime}$ has $F C$.

Proof. It clearly suffices to consider the case where $R^{\prime}=R[\xi]$ is a simple $R$-algebra. ${ }^{2}$ Moreover, since FC is preserved under homomorphic image, we may assume $R[\xi]=R[X] /(f(X))$, where $f(X)$ is a monic polynomial. Suppose there exists an ideal $\mathfrak{a}^{\prime}$ in $R^{\prime}$ having an infinite number of minimal primes, say $\left\{P_{\alpha}^{\prime}\right\}$. We may assume that $\mathfrak{a}^{\prime}=\bigcap_{\alpha} P_{\alpha}^{\prime}$. Since $R$ has $\mathrm{FC}, \mathfrak{a}^{\prime} \cap R=\mathfrak{a}$ has only finitely many minimal primes, so there must exist a minimal prime $P_{1}$ of a such that $P_{1}$ is contained in infinitely many of the $P_{\alpha}^{\prime}$. Since $R^{\prime}=R[X] /(f(X))$ is a finite free $R$-module every minimal

Received by the editors October 24, 1972.

AMS (MOS) subject classifications (1970). Primary 13A15, 13B20, 13E05, $13 \mathrm{G} 05$.

Key words and phrases. Minimal primes of an ideal, integral ring extension, noetherian integral domain, derived normal ring, noetherian prime spectrum.

1 The author received partial support for this research from National Science Foundation grant GP-29326.

2 It is not true in general that $R$ having $\mathrm{FC}$ implies the polynomial ring $R[X]$ has FC [3, Example 2.9, p. 635].

c) American Mathematical Society 1973 
prime of $P_{1} R^{\prime}$ lies over $P_{1}$ in $R$ and $P_{1} R^{\prime}$ has only finitely many minimal primes. It follows that some minimal prime $P_{1}^{\prime}$ of $P_{1} R^{\prime}$ is contained in infinitely many of the $P_{\alpha}^{\prime}$. Let $a_{1}^{\prime}=\bigcap\left\{P_{\alpha}^{\prime} \mid P_{1}^{\prime} \subset P_{\alpha}^{\prime}\right\}$. The fact that $P_{1}^{\prime}$ is contained in infinitely many of the $P_{\alpha}^{\prime}$ implies that $\mathfrak{a}^{\prime}$ is not contained in $P_{1}^{\prime}$ and $P_{1}^{\prime}$ is properly contained in $\mathfrak{a}_{1}^{\prime}$. Thus $\mathfrak{a}_{1}^{\prime}$ has infinitely many minimal primes and $a_{1}^{\prime} \cap R=a_{1}$ properly contains $P_{1}$. We note also that some minimal prime of $P_{1} R^{\prime}$ is in the set $\left\{P_{\alpha}^{\prime}\right\}$. For $P_{1}$ occurs in a representation of $a=a^{\prime} \cap R$ as a finite irredundant intersection of prime ideals. Hence if $\mathfrak{b}^{\prime}=\bigcap\left\{P_{\alpha}^{\prime} \mid P_{1} \subset P_{\alpha}^{\prime}\right\}$, then $\mathfrak{b}^{\prime} \cap R=P_{1}$. Since $P_{1} R^{\prime}$ has only finitely many minimal primes, it follows that some $P_{\alpha}^{\prime}$ is a minimal prime of $P_{1} R^{\prime}$. Proceeding now in this manner with $\mathfrak{a}_{1}^{\prime}$, we can construct for any positive integer $n$, a chain $P_{1}<\cdots<P_{n}$ of prime ideals in $R$ such that each $P_{i}$ is the contraction of some prime in the set $\left\{P_{\alpha}^{\prime}\right\}$. By choosing $n>\operatorname{deg} f=m$, we show that this leads to a contradiction. We may assume that $P_{1}=(0)$, and hence that $R$ is an integral domain. Let $T$ be the integral closure of $R$ in an algebraic closure of the quotient field of $R$ and let $(0)=Q_{1}<\cdots<$ $Q_{n}$ be primes of $T$ such that $Q_{i} \cap R=P_{i}$. Let $T[\xi]=T[X] /(f(X))=$ $T \otimes_{R} R[\xi]$ and identify $T$ and $R[\xi]$ as subrings of $T \otimes_{R} R[\xi], T=T \otimes 1$ and $R[\xi]=1 \otimes R[\xi]$. In $T[X]$ we have $f(X)=\left(X-\xi_{1}\right) \cdots\left(X-\xi_{m}\right)$. Let $Q_{i j}^{\prime}$ denote the prime in $T[\xi]$ lying over $Q_{i}$ in $T$ and corresponding to the root $\xi_{j}$ of $f(X)$. Of course not all the $Q_{i j}^{\prime}, 1 \leqq j \leqq m$, need be distinct, but we have $Q_{1 j}^{\prime}<Q_{2 j}^{\prime}<\cdots<Q_{n j}^{\prime}$. Moreover, if $Q_{i j}^{\prime} \cap R[\xi]=P_{i j}^{\prime}$, then $\left\{P_{i j}^{\prime}\right\}_{j=1}^{m}$ is the set of minimal primes of $P_{i} R[\xi]$ and $P_{1 j}^{\prime}<P_{2 j}^{\prime}<\cdots<P_{n j}^{\prime}$. But by our construction, for each $i$, some $P_{i j}^{\prime} \in\left\{P_{\alpha}^{\prime}\right\}$. Since there are no containment relations among the elements of $\left\{P_{\alpha}^{\prime}\right\}$, this implies that $n \leqq m$ and completes the proof. Q.E.D.

A ring $R$ is said to have noetherian spectrum if the radical ideals in $R$ satisfy the ascending chain condition. Conditions equivalent to $R$ having noetherian spectrum are that $R$ have FC and satisfy the ascending chain condition on prime ideals.

If $R \subset R^{\prime}$ are integral domains, then $R^{\prime}$ is said to be almost finite over $R$ if $R^{\prime}$ is integral over $R$ and if the quotient field of $R^{\prime}$ is a finite algebraic extension of the quotient field of $R$ [2, p. 30].

THEOREM 2. If $R$ is a noetherian integral domain and $R^{\prime}$ is an almost finite extension of $R$, then $R^{\prime}$ has noetherian spectrum.

Proof. It will suffice to consider the case when $R^{\prime}$ is integrally closed, for if the integral closure of $R^{\prime}$ has noetherian spectrum then so does $R^{\prime}$. We first show that, for any ideal $a$ in $R, a R^{\prime}$ has only a finite number of minimal primes. We proceed by induction on the number of generators for a. Principal ideals in $R^{\prime}$ have only finitely many minimal primes, for $R^{\prime}$ is the derived normal ring of a noetherian domain and hence is a 
Krull domain [2, p. 118]. If $\mathrm{a}=\left(x_{1}, \cdots, x_{n}\right)$, let $P_{1}^{\prime}, \cdots, P_{m}^{\prime}$ be the minimal primes of $x_{1} R^{\prime}$. Then $R^{\prime} \mid P_{i}^{\prime}$ is an almost finite extension of $R /\left(P_{i}^{\prime} \cap R\right)\left[2\right.$, p. 118], and by our induction assumption $\mathrm{a}\left(R^{\prime} \mid P_{i}^{\prime}\right)$ has only finitely many minimal primes. Since every minimal prime of a $R^{\prime}$ contains at least one of the $P_{i}^{\prime}$, it follows that $a R^{\prime}$ has only finitely many minimal primes. To complete the proof of the theorem, we can now proceed as as in Theorem 1, viz. if $a^{\prime}$, were an ideal in $R^{\prime}$ having infinitely many minimal primes then we could construct in $R$ an infinite strictly ascending chain $P_{1}<P_{2}<\cdots$ of prime ideals. This would of course contradict the fact that $R$ is noetherian.

COROLlaRY. If $R$ is a noetherian integral domain, then the derived normal ring of $R$ has noetherian spectrum.

In connection with properties of the derived normal ring of a noetherian integral domain, we would like to ask the following.

Question. If $R$ is a noetherian integral domain with integral closure $\bar{R}$, must it follow that maximal ideals in $\bar{R}$ are finitely generated?

In trying to show this to be true, a simple induction argument on the Krull dimension of $R$ runs into the difficulty that there can exist between a 2-dimensional noetherian domain $A$ and the integral closure of $A$ a ring $B$ having a non-finitely-generated maximal ideal. To get an example illustrating this one can use the following construction suggested to me by Kaplansky. Let $T$ be a 1-dimensional local (noetherian) domain such that the integral closure of $T$ is not a finite $T$-module. Let $T<T_{1}<T_{2} \cdots$ be a strictly ascending chain of finite $T$-algebras between $T$ and the integral closure of $T$. Let $X$ be an indeterminate and let $A=T[X]$, and $B=T+$ $T_{1} X+T_{2} X^{2}+\cdots$. Then $B$ is a ring between $A$ and the integral closure of $A$ and if $m$ is the maximal ideal in $T$, then $m+T_{1} X+T_{2} X^{2} \cdots$ is a nonfinitely-generated maximal ideal in $B$.

\section{REFERENCES}

1. W. Heinzer, A note on rings with noetherian spectrum, Duke Math. J. 38 (1970), 573-578. MR 41 \#8395.

2. M. Nagata, Local rings, Interscience Tracts in Pure and Appl. Math., no. 13, Interscience, New York, 1962. MR 27 \#5790.

3. J. Ohm and R. Pendleton, Rings with noetherian spectrum, Duke Math. J. 35 (1968), 631-639. MR 37 \#201.

Department of Mathematics, Purdue University, Lafayette, Indiana 47907 